\title{
Clinical Observation of UreTron Single-Probe Ultrasonic Intracorporeal Lithotripter for Ureteral Calculi
}

\author{
Peng Zhang \\ Xiu-Wu Han \\ Xin Zhang \\ Xu-Hui Zhu \\ Tao Li \\ Yan-Sheng Li \\ Yuan-Hao Chen \\ Gao Li
}

Department of Urology, Beijing ChaoYang Hospital, Beijing, People's Republic of China
Correspondence: Xiu-Wu Han Department of Urology, Beijing ChaoYang Hospital, Capital Medical University, 5 Jingyuan Road, Shijingshan District, Beijing, People's Republic of China Tel +86 I0-13501356960

Email hanxiuwu47859@163.com
Objective: The present study aimed to observe and discuss the effectiveness and safety of the UreTron single-probe ultrasonic intracorporeal lithotripter in ureteroscopic lithotripsy.

Methods: The clinical data of patients with unilateral solitary ureteral calculi treated with ureteroscopic lithotripsy who were hospitalized at the Department of Urology (West District) of Beijing Chaoyang Hospital between March 2016 and August 2020 were selected for retrospective analysis. The patients were divided into the UreTron group (group U) and holmium laser group (group H) according to the lithotripsy method adopted. The operation duration, length of hospital stay, stone clearance rate, proportion of patients requiring flexible ureteroscopy-assisted lithotripsy, and complications were compared between the groups.

Results: There was no statistical difference between the groups in terms of the general characteristics, operation duration, or length of hospital stay $(\mathrm{P}>0.05)$. Regarding the stone clearance rate (group $\mathrm{U}=93.5 \%$; group $\mathrm{H}=75 \%$ ), proportion of patients requiring flexible ureteroscopy-assisted lithotripsy (group $\mathrm{U}=6.5 \%$; group $\mathrm{H}=27.8 \%$ ), and incidence of surgical complications (group $\mathrm{U}=1$ case; group $\mathrm{H}=9$ cases), group $\mathrm{U}$ was superior to group $\mathrm{H}$, and the differences between the groups were statistically significant $(\mathrm{P}<0.05)$. However, the differences in other complications (cardiocerebral complications and lower extremity thrombosis) were not statistically significant between the groups $(\mathrm{P}>0.05)$.

Conclusion: The UreTron system is a new lithotripsy apparatus that is safe and effective for ureteroscopic lithotripsy and has certain advantages in terms of the stone clearance rate, proportion of patients requiring flexible ureteroscopy-assisted lithotripsy, and surgical complications, making it worthy of clinical application.

Keywords: UreTron, holmium laser, ureteral calculi, lithotripsy

\section{Introduction}

Urolithiasis is a common urologic disease with a prevalence of approximately $2-3 \%$ in the general population, and the incidence and recurrence rates are gradually increasing worldwide. In recent years, the incidence of urolithiasis in China has reached approximately $6.5 \%$, while in the United States, it has reached as high as $10 \%$. It is correlated with genetics, environment, and diet among other factors. Urolithiasis is also a major reason for emergency visits to urology departments as well as hospitalizations, causing a huge health and economic burden on patients. ${ }^{1-3}$ Ureteral calculi are often treated surgically because of their tendency to cause acute pain, chronic obstruction and hydronephrosis, and renal function loss. Recently, stone removal by ureteroscopic lithotripsy has been adopted in most cases, ${ }^{3}$ and holmium laser apparatus are most 
commonly used. ${ }^{4,5}$ However, the problems of stone displacement, ureteral mucosal injury, and even distant ureteral stricture caused by this procedure are still a great challenge in clinical practice. ${ }^{6,7}$ The new UreTron single-probe ultrasonic intracorporeal lithotripter (Med-Sonics) was introduced to our department in 2015. In this study, the clinical results of ureteroscopic lithotripsy with this system between March 2016 and August 2020 are analyzed, and the results are compared with those of patients who underwent holmium laser lithotripsy to evaluate the UreTron system's effects and safety.

\section{Materials and Methods Data of Clinical Cases}

The clinical data of patients with ureteral calculi treated with ureteroscopic lithotripsy who were hospitalized at the Department of Urology (West District) of Beijing Chaoyang Hospital between March 2016 and August 2020 were collected. A retrospective study was conducted on the above data. The inclusion criteria were as follows: patients with a single, solitary stone located in the unilateral ureter, as confirmed by imaging results (ultrasound, kidney, ureter, and bladder [KUB] X-ray, or computed tomography [CT]); patients with $7 \mathrm{~mm} \leq$ stone diameter $\leq 20 \mathrm{~mm}$ (calculi size was assessed based on CT scan in all cases); and patients eligible for ureteroscopic lithotripsy. All cases met the inclusion criteria were assigned to either group $\mathrm{U}$ or group $\mathrm{H}$ according to the principle of "complete randomization". The exclusion criteria were as follows: patients with a history or diagnosis of urinary stone attack, lithotripsy, or surgery-related medical problems within six months before surgery; patients with multiple stones or combined with renal calculi; patients with stones less than $7 \mathrm{~mm}$ or greater than $20 \mathrm{~mm}$ in diameter; patients with a definite preoperative urinary tract infection (according to the urine routine and urine culture examination results) that had not been corrected; patients with severe ureteral stricture found intraoperatively who were unable to complete the first stage of lithotripsy; and patients with cardiac and pulmonary insufficiency, renal insufficiency, severe hemorrhagic tendency or disease, severe lumbar spine or hip lesions unsuitable for the lithotomy position, and/or comorbidities or contraindications unsuitable for surgery. The cases meeting the criteria were divided into the UreTron group (group U; $\mathrm{N}=31$ cases) and holmium laser group (group $\mathrm{H} ; \mathrm{N}=36$ cases) according to the system adopted.

\section{Lithotripsy Apparatus}

UreTron (Med-Sonics, Erie, PA, America)

Single-Probe Ultrasonic Intracorporeal Lithotripter

The apparatus (Figure 1) used was a new single-handle, single-probe lithotripsy system with three functions: ultrasonic lithotripsy, "similar" ballistic lithotripsy, and negative pressure suction on a single lithotripter rod. It is called the "similar" ballistic lithotripsy because it differs from previous ballistic lithotripsy equipment via adjustment of the mechanical force or amplitude to achieve lithotripsy through special technology using a different ultrasound frequency. Through unique electric-kinetic energy conversion and alongside the ultrasound action, the vibration along the probe catheter is transmitted to the tip to produce an axial motion with a length of $20-100 \mu \mathrm{m}$ to optimally distribute the energy when acting on the stones. The change in frequency achieves the effect of impact, and in a short time, the stone disintegrates and is sucked out because it cannot withstand the applied energy (Figure 2). The vibration frequency can reach $21,000 \pm$ $1000 \mathrm{HZ}$, similar to other lithotripsy apparatus. The apparatus is available with rigid, semi-flexible, and flexible probes to suit different surgical requirements. The probe rod suitable for the ureteroscopic lithotripsy adopted in the present study was semi-flexible (with an outside diameter of $1.6 \mathrm{~mm} \times 56 \mathrm{~cm}$ ). The excitation modes during the surgery were divided into two types: soft and hard.

\section{LISA Holmium Laser Lithotripsy Apparatus}

The LISA holmium laser (Sphinx, 60W, Germany) was used. A 272-micron fiber was adopted in the surgery, with parameters of $0.8 \sim 1.0$ joule for the pulse energy, $20 \mathrm{~Hz}$ for the frequency, and 200 microns for the pulse width.

\section{Surgical Methods}

All surgeries were performed by the same surgeon, and the assistants were from the same treatment team. Conventional tracheal intubation or laryngeal mask general anesthesia was adopted, with the patients in the lithotomy position. A WOLF F8/9.8 ureteroscope was used to enter the bladder under direct vision, and a super-slip guidewire (Boston Scientific, ZIP wire) was placed retrograde into the affected ureter under ureteroscopic observation with the patient in a head-high, foot-low position. The ureteroscope was then inserted forward under the guidance of the guidewire. The UreTron or holmium laser apparatus was used for the lithotripsy to find the calculi, with the powder from the UreTron lithotripsy aspirated into 


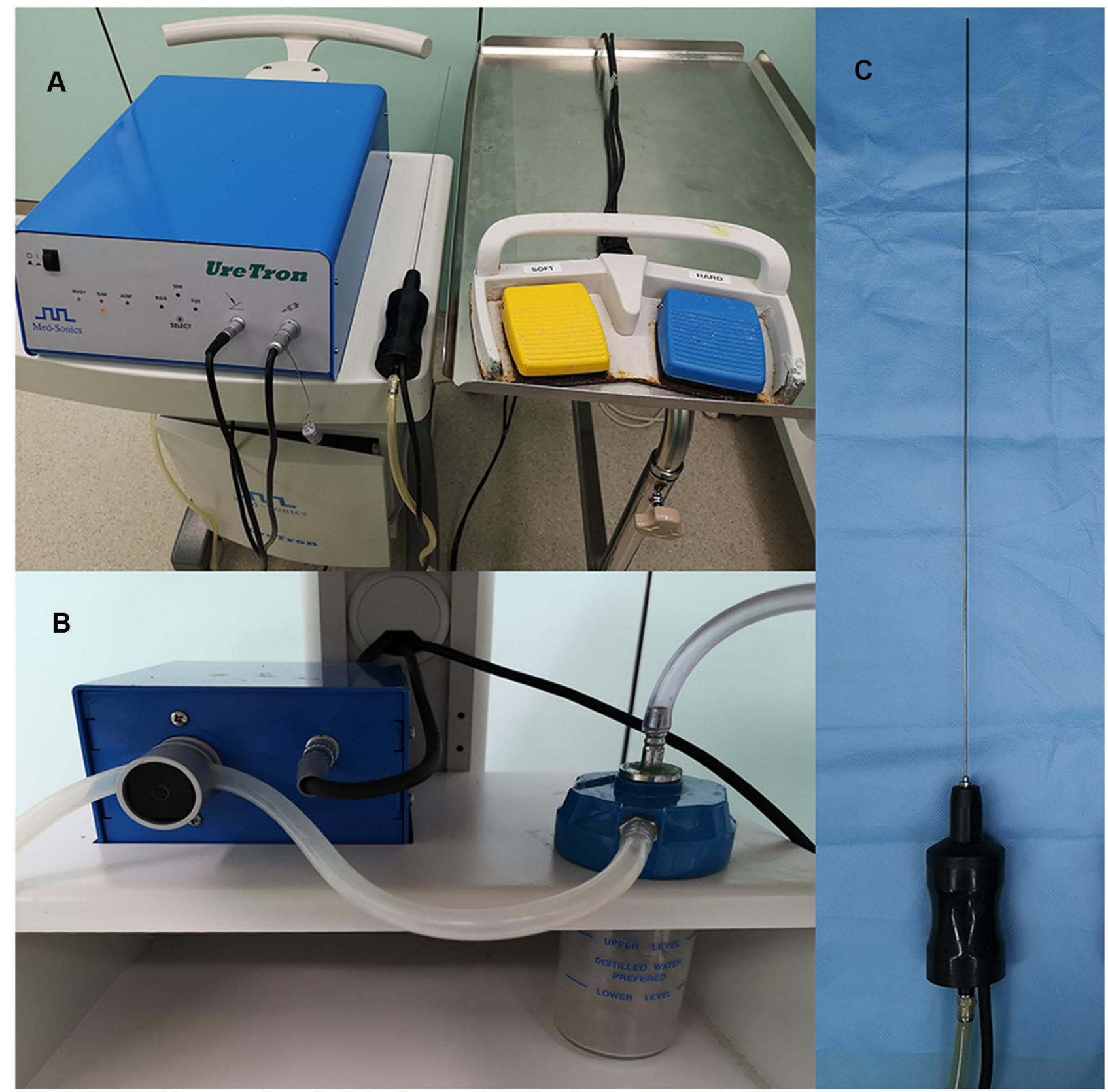

Figure I The UreTron single probe ultrasonic intracorporeal lithotripsy system. ((A) Equipment composition and connection status; (B) Negative pressure drainage and stone powder recovery device; (C) The single-handle, single probe lithotripsy rod suitable for ureteroscopic lithotripsy).

a collection bottle. An interceptor basket (Boston Scientific, Stone Cone) was used to block the proximal displacement of the stone as appropriate. In the case of stone displacement into the pelvis and calyces, which prevented further lithotripsy, a flexible ureteroscope (POLYDIAGNOST or Olympus URF-V) was adopted to assist (all with the holmium laser apparatus), and a mesh basket (Boston Scientific, Zero TIP) was used as appropriate. After the lithotripsy, a ureteral stent tube (Boston Scientific, Polaris Loop, $6 \mathrm{~F} \times 24 \mathrm{~cm}$ ) was routinely placed and retained for four weeks, and a urinary catheter was placed and retained for three days.

\section{Evaluation and Judgment Methods}

\section{Evaluation Indicators}

The differences in the operation duration, length of hospital stay, stone clearance rate, proportion of patients requiring flexible ureteroscopy-assisted lithotripsy, surgical complications (fever, ureteral stricture, and steinstrasse formation), and other complications 


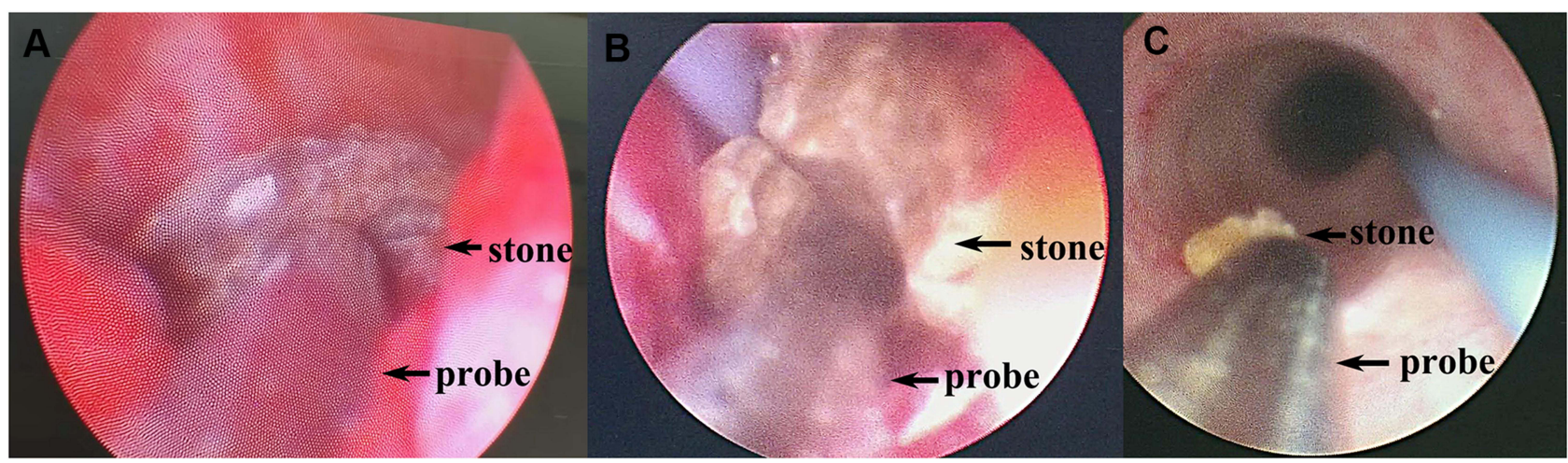

Figure 2 The intraoperative lithotripsy process with the UreTron single probe ultrasonic lithotripsy system. ((A and B) The Single-handle, single probe provided both ultrasound and ballistic lithotripsy performance intraoperatively; (C) Removal of crushed stone fragments by negative pressure suction).

(cardiocerebral complications and deep vein thrombosis [DVT]) were compared between the two groups.

\section{Judgment Methods}

Operation duration: The timing started once the ureteroscope was successfully inserted into the urethra and terminated when the ureteral stent tube was inserted upon completion of the lithotripsy. If ureteroscopy-assisted lithotripsy was required, the time consumed was also included (in minutes).

Stone clearance rate: The KUB or CT was re-examined within three days after the surgery. A lack of residual stones, stone fragments $\leq 2.5 \mathrm{~mm}$, and clinical symptoms was defined as stone clearance.

Fever: a temperature above $38^{\circ} \mathrm{C}$ within three days after surgery and requiring clinical management was defined as fever.

Ureteral stricture: Non-stone obstructive hydronephrosis or simple ureteral stricture on the operated side identified by enhanced CT examination within six months after the surgery and confirmed by re-operation or therapeutic ureteroscopy was defined as ureteral stricture.

\section{Statistical Methods}

The IBM SPSS Statistics 22.0 software was adopted for the data analysis. The measurement data were expressed as the mean \pm standard deviations $(\mathrm{x} \pm \mathrm{s})$ and compared by a $t$-test. The countable data were expressed as rates (\%), and the chi-square test was adopted for comparison. A P-value $<0.05$ was considered statistically significant.

\section{Results}

\section{Comparison of the General}

\section{Characteristics Between the Two Groups}

\section{(Table I)}

There was no statistical difference between the groups in terms of the ratio of gender, age, body mass index, distribution of ureteral calculi (right or left and upper, middle, or lower segments), or stone size ( $\mathrm{P}>0.05)$, indicating that the surgical data were comparable between the groups.

\section{Comparison of the Indicators Relevant to Surgery and Complications Between the Two Groups (Tables 2 and 3)}

There was no statistical difference between the groups in terms of the operation duration or length of hospital stay $(\mathrm{P}>0.05)$. In terms of the stone clearance rate (group $\mathrm{U}=93.5 \%$; group $\mathrm{H}=75 \%$ ), proportion of patients requiring flexible ureteroscopy-assisted lithotripsy (group $\mathrm{U}=6.5 \%$;

Table I Comparison of General Information Between the Two Groups

\begin{tabular}{|c|c|c|c|c|c|c|c|}
\hline & $\begin{array}{c}\text { Number } \\
\text { of Cases }\end{array}$ & $\begin{array}{c}\text { Gender (M/ } \\
\text { F) (Case) }\end{array}$ & Age (Years) & BMI & $\begin{array}{c}\text { \#Left/Right } \\
\text { (Case) }\end{array}$ & $\begin{array}{c}\text { \#Upper/Middle-Lower } \\
\text { Segment (Case) }\end{array}$ & $\begin{array}{c}\text { The Stone } \\
\text { Diameter (mm) }\end{array}$ \\
\hline U Group & 31 & $19 / 12$ & $45.48 \pm 13.30$ & $26.50 \pm 3.40$ & $18 / 13$ & $11 / 20$ & $12.40 \pm 2.75$ \\
\hline H Group & 36 & $23 / 13$ & $41.19 \pm 11.23$ & $27.06 \pm 3.81$ & $19 / 17$ & $17 / 19$ & $13.09 \pm 2.86$ \\
$\quad t$ value & - & - & 1.431 & -0.631 & - & - & -1.010 \\
$X^{2}$ value & - & 0.048 & - & - & 0.188 & 0.943 & - \\
P value & - & 0.826 & 0.157 & 0.530 & 0.664 & 0.331 & 0.316 \\
\hline
\end{tabular}

Note: ${ }^{\#}$ The location of the calculi in the ureter.

Abbreviation: BMI, Body Mass Index. 
Table 2 Comparison of the Operation-Related Indicators Between the Two Groups

\begin{tabular}{|l|c|c|c|c|}
\hline & $\begin{array}{c}\text { The Operation } \\
\text { Duration (Min) }\end{array}$ & $\begin{array}{c}\text { The Length of } \\
\text { Hospital Stay (Day) }\end{array}$ & $\begin{array}{c}\text { The Stone } \\
\text { Clearance Rate } \\
\text { (\%) }\end{array}$ & $\begin{array}{c}\text { Those Need the Flexible Ureteroscopy- } \\
\text { Assisted Lithotripsy (Case) }\end{array}$ \\
\hline U Group & $60.52 \pm 14.16$ & $7.87 \pm 1.86$ & $29(93.5 \%)$ & $2(6.5 \%)$ \\
\hline H Group & $66.94 \pm 16.40$ & $8.69 \pm 2.03$ & $27(75 \%)$ & $10(27.8 \%)$ \\
$t$ value & -1.703 & -1.724 & - & - \\
$X^{2}$ value & - & - & 4.176 & 5.153 \\
P value & 0.093 & 0.09 & $0.041^{*}$ & $0.023^{*}$ \\
\hline
\end{tabular}

Note: $* \mathrm{P}<0.05$.

Table 3 Comparison of the Postoperative Complications Between the Two Groups

\begin{tabular}{|c|c|c|c|c|c|c|c|}
\hline & \multicolumn{2}{|c|}{ The Operation-Related Complications (Case) } & \multicolumn{3}{c|}{ Other Complications (Case) } \\
\cline { 2 - 7 } & Fever & $\begin{array}{c}\text { Ureteral } \\
\text { Stricture }\end{array}$ & $\begin{array}{c}\text { Steinstrasse } \\
\text { Formation }\end{array}$ & Total & $\begin{array}{c}\text { Cardiocerebral } \\
\text { Complications }\end{array}$ & $\begin{array}{c}\text { Lower Extremity } \\
\text { Thrombosis }\end{array}$ & Total \\
\hline U Group & $\mathrm{I}$ & 0 & 0 & $\mathrm{I}$ & $\mathrm{I}$ & 0 & $\mathrm{I}$ \\
\hline $\mathrm{H}$ Group & 5 & 2 & 2 & 9 & 0 & - & - \\
$\quad \begin{array}{r}\text { value } \\
X^{2} \text { value }\end{array}$ & - & - & - & - & - & - & - \\
P value & 0.273 & 0.495 & - & 4.623 & - & 1.000 & 1.000 \\
\hline
\end{tabular}

Notes: $* \mathrm{P}<0.05$; Cardiovascular complications as postoperative atrial fibrillation; Lower extremity thrombosis is lower extremity peroneal vein thrombosis.

group $\mathrm{H}=27.8 \%$ ), and incidence of surgical complications (group $\mathrm{U}=1$ case; group $\mathrm{H}=9$ cases), group $\mathrm{U}$ was superior to group $\mathrm{H}$, and the differences between the groups were statistically significant $(\mathrm{P}<0.05)$. However, the differences in other complications (Cardiocerebral complications and Lower extremity thrombosis) were not statistically significant between the groups $(\mathrm{P}>0.05)$.

\section{Equipment Failure Conditions}

In addition to the observation indicators mentioned above, we also recorded the failures of the two lithotripsy apparatus during the study. Regarding the holmium laser apparatus, three failures occurred, two of which were due to laser fiber breakage (solved by replacing the fiber with a new one) and one to laser generator overheating (recovered after suspending operation and turning off the apparatus). In group $U$, two failures occurred, one of which was due to lithotripsy rod breakage (solved by replacing the rod with a new one) and the other to lithotripsy rod blockage by stone powder (solved by unblocking and flushing). Considering that fracture of optic fiber and lithotripsy rods is correlated with the number of applications, degree of wear and tear, and operation methods and are all normal wear and tear apparatus, it is difficult to evaluate scientifically. Therefore, these indicators were not included in the analysis in the present study and were provided for clinical reference only. In addition, other reports have mentioned that the failure rate of the UreTron apparatus is not significantly different from that of other equipment and that the main cause of failure is blockage of the probe rod. ${ }^{8}$

\section{Discussion}

Minimally invasive surgery has long been the primary means of treating urinary stones, and the main procedure is endoscopic surgery through natural anatomical channels, eg, ureteroscopy, percutaneous nephroscopy, and cystoscopy, which are characterized by less injury and hemorrhage and higher safety and effectiveness. However, an ideal lithotripsy system is a prerequisite for the successful treatment of stones with the above surgical procedures. From the early electrohydraulic lithotripsy apparatus to the later widely used pneumatic ballistic, ultrasound, ultrasound combined with ballistic, and holmium laser lithotripsy apparatus, the search for an optimal lithotripsy system has long been pursued. Thus far, all abovementioned lithotripsy systems have their own advantages and disadvantages. 
In recent years, a new type of lithotripsy "tool," the UreTron, has been gradually introduced to clinical practice. Its main features are the combination of ultrasound, ballistic lithotripsy, and negative pressure suction as well as its creative single-handle, single-probe apparatus connection. The system has many advantages, including highefficiency lithotripsy, negative pressure suction, and convenient operation. In a study conducted by Borofsky et $\mathrm{al}^{8}{ }^{8}$ the advantages of the UreTron over several similar lithotripsy systems currently in use for percutaneous nephrolithotomy, including the CyberWand (single-handle, dualprobe), StoneBreaker, and LithoClast Select, were reported. The UreTron was considered a safer and more effective lithotripsy system because of its higher lithotripsy efficiency, especially for difficult-to-handle stones, and no significant difference in complications or apparatus failure was found. In the present study, we focused on the performance of the UreTron in ureteroscopic lithotripsy and compared the results with those of the holmium laser lithotripsy system most commonly used for this operation.

It could be inferred from the results that group $\mathrm{U}$ maintained the same results as group $\mathrm{H}$ in terms of the operation duration and length of hospital stay. Regarding other complications not directly correlated with the operation, group $\mathrm{U}$ also maintained the same low level as group $\mathrm{H}$, with postoperative arrhythmia and lower-extremity DVT being the most common complications after general anesthesia and lithotomy position. There was no evidence of a direct relationship with the lithotripsy device or modality. The patients who developed these complications were discharged after improvement with no adverse consequences after receiving general symptomatic treatment. These results demonstrated the efficiency and safety of UreTron in surgical applications.

In comparing another group of indicators, it was found that group $U$ had advantages in the stone clearance rate, proportion of patients requiring flexible ureteroscopyassisted lithotripsy, and complications directly correlated with the operation, which are precisely what urologists focus on during ureterolithotripsy.

Intraoperative stone displacement, especially that of stones in the upper and middle segments, can lead to an inability to complete lithotripsy or the need to change to flexible ureteroscopy assisted lithotripsy, resulting in increased operation duration, cost, and possible complications, which often disturb the specialist. In the observation of the relevant indicators, it was found that stone displacement into the pelvic calyces and the need for ureteroscopy- assisted lithotripsy in both groups occurred primarily during the process of lithotripsy. Due to the need for uninterrupted intraoperative flushing with normal saline to avoid the stone powder affecting the clarity of the surgical field as well as the need to increase the water flow or pressure, stone displacement can occur. In particular, stones are more likely to be displaced by water flow after partial comminution, reducing in size, loosening from the stone bed, or falling off of larger stone fragments. Although we took measures to prevent stone displacement via a head-up, feet-down position, dynamic control of water pressure and flow, and the use of interceptor baskets as appropriate, it was still difficult to avoid the occurrence of stone displacement completely. Particularly in cases with significant proximal ureteral dilatation, even with an interceptor basket, the dilated ureteral lumen was not completely covered, and smaller stone fragments were more likely to pass through the lateral part of the basket or orifice, where they were displaced by the constant water impact and pressure. ${ }^{9,10}$ In the observation of the above cases, it was found that group $\mathrm{U}$ showed less stone displacement, mainly due to the high-frequency ultrasonic vibration adsorption effect of the system and the negative pressure suction. The former reduced the stone fragmentation and disintegration, and the latter prevented the stones from drifting with the rinsing fluid to achieve suction while fighting, fragmentation and clearing in one step.

The stone clearance rate is an important indicator to evaluate lithotripsy outcomes. The present study focused on the lithotripsy results within three days after the surgery to assess the intraoperative lithotripsy ability of the UreTron apparatus. As the ureteroscopic approach itself has a high stone clearance rate, a strict index was adopted to define the meaning of this rate (no remaining stones larger than $2.5 \mathrm{~mm}$ in diameter). We found that the failure to meet the above requirements was basically related to the residual caused by the failure to powder part of the calculi. The residual stones generally occurred after the conversion to flexible ureteroscopy due to the inability to powder some of the stones and completely remove the fragmented stones. Particularly in group $\mathrm{H}$, the stage I clearance rate was slightly lower than the approximately $80 \%$ reported in other literature works. ${ }^{11,12}$ This may have been due to the stricter criteria for residual stones defined in the present study and the fact that stone fragments drifted into the kidneys in dispersed locations and were more difficult to retrieve. Therefore, in addition to reducing the incidence of stone displacement and residual stone fragmentation, 
the previously described characteristics of "suction while fighting, fragmentation and clearing in one step" in group $\mathrm{U}$ were also advantageous in terms of the stone clearance rate due to the lower conversion rate of flexible ureteroscopy. However, during the follow-up, it was found that many cases did not reach the stone clearance standard within the three days after the operation. Most of the small stone fragments had expelled by themselves by the re-examination, with the ureteral stent tube being placed until one month post-operation.

In terms of the complications directly correlated with the operation, both treatment groups showed lower incidences of fever, ureteral stricture, and steinstrasse formation. When the incidences of these three complications were combined and compared between the groups, group $\mathrm{U}$ showed a lower incidence, which further illustrated the advantages of the UreTron. Although the intergroup comparisons of the individual indicators also showed that group $U$ fared better, the differences were not statistically significant between the groups, which might be correlated with the small sample size. Since these three indicators are important aspects in evaluating the outcome of the operation and safety of the lithotripsy apparatus, they were analyzed separately based on actual clinical observations.

The main cause of postoperative fever and urogenic sepsis is the entry of bacteria into the bloodstream through damaged mucosal barriers or blood vessels; these complication are directly correlated with intrapelvic hypertension, duration, and perfusion rate. ${ }^{13-15}$ Fever is more frequent after flexible ureteroscopic and percutaneous nephrological procedures than after hard ureteroscopic procedures. The intraoperative low-pressure flushing guaranteed by the negative pressure suction effect of the UreTron system as well as the lower conversion rate to flexible ureteroscopy might have contributed to the lower number of cases with postoperative fever in group $U$.

Physical or thermal damage to the ureteral mucosa and wall caused by the laser energy during holmium laser lithotripsy is difficult to avoid completely and is the possible factor of ureteral stricture after this procedure. The two cases of postoperative ureteral stricture observed in the present study were both in group H. Physical injuries range from minor mucosal burns to perforations and lacerations of varying severity that can result in corresponding adverse outcomes. Another more insidious and unavoidable mechanism of injury is the thermal damage caused by the laser energy output, ${ }^{16,17}$ which is also an undetectable cause of postoperative ureteral stricture. With the full reorganization of this possible hazard, it can be avoided by controlling the laser output energy, reducing the operation duration, increasing the flow rate of the rinse fluid, and controlling the temperature of the rinse fluid; however, ureteral injury and stricture caused by the thermal effect remains a difficulty of laser lithotripsy. Refractory ureteral stricture is also a major problem for specialists. Therapeutic methods such as stent placement and drainage, balloon dilation, resection and reanastomosis of the stenotic segment, and ureteral replacement therapy can cause varying degrees of physical damage and financial burden. Of the two patients with ureteral stricture in the present study, one was cured by ureteroscopic dilatation, replacement, and extended ureteral stent placement until six months post-operation; the other was treated with Allium stent placement. Ultrasonic energy was adopted in the UreTron lithotripsy system to achieve lithotripsy and fundamentally avoid the "boiled ureter phenomenon" caused by the thermal effect. The high-frequency vibration of ultrasound only acts on the stone and does not damage the soft tissue of the ureteral mucosa, thus greatly avoiding complications such as burns and perforations and probably reducing the incidence of postoperative ureteral stricture. There were no cases of postoperative ureteral stricture in group U.

Postoperative steinstrasse formation is uncommon after ureteral lithotripsy and occurs mostly when larger stones are treated by flexible ureteroscopy. ${ }^{18,19}$ In the two cases of steinstrasse formation in group $\mathrm{H}$ observed in the present study, both were large-diameter hard stones that could not be powdered by holmium laser lithotripsy, resulting in a large number of small fragments that were not removed intraoperatively, resulting in postoperative steinstrasse formation. The patients were given extracorporeal shock wave lithotripsy and routine lithotripsy treatment with the postoperative placement of a ureteral stent, and the stones were expelled within one month without any adverse consequences. In group $U$, steinstrasse formation was difficult because of the effects of the ballistic lithotripsy, which could break up the harder stones, and ultrasonic lithotripsy, which could further powder and remove stone fragments simultaneously.

Some initial insight was gained during the operations in the present study. For hard stones, especially those with a hard shell, the UreTron system had difficulty in the initial stage of lithotripsy. Gently pushing the stone and alternately rotating the handle rapidly in both directions (similar to a grinding motion) could speed up the 
lithotripsy process, and once the shell was broken or the stone fragmented, subsequent lithotripsy became easier. During lithotripsy with the adoption of UreTron, due to the small space of the ureteral lumen, negative pressure suction during the procedure may cause lumen closure due to the negative pressure, which may affect the observation of stones and cause adsorption of the mucosa on the tip of the probe rod, interfering with the lithotripsy and causing mucosal edema. Although these conditions do not result in substantial injury, they can cause some distress. In our experience, while the operator was performing lithotripsy, the assistant had to adjust the suction pressure by partially clamping the negative pressure suction tube or intermittently opening and closing it by clamping to effectively suck the stone powder without affecting the stone observation or continuous lithotripsy operation. In addition, the assistant could also unclamp the stone powder in the drainage tube by clamping, opening/closing, or squeezing to reduce the possibility of probe rod blockage. Moreover, it was observed that before the lithotripsy probe rod broke, the lithotripsy efficiency was often weakened and the root of the probe rod turned black due to wear and tear; thus, the probe rod should be actively replaced. During lithotripsy with the probe rod, maintaining the rod's axial level and avoiding bending or angulation with the long axis of the ureteroscopy prolonged the rod's use.

The present study was only a preliminary study of the UreTron apparatus in ureteroscopic lithotripsy, with a small sample size and no comparison with other lithotripsy apparatus and procedures. In future studies, we will expand the sample size and compare different surgical modalities, eg, percutaneous nephrolithotripsy, ureteral flexible lithotripsy, and cystoscopy lithotripsy, and other commonly used lithotripsy apparatus, eg, the ultrasonic ballistic lithotripsy system, to comprehensively and objectively evaluate the efficacy of the UreTron system in various stone operations.

There were some limitations in this study. This study is a retrospective study which has the risk of confounding by an unmeasured coefficient, this potential bias is a limitation.

\section{Conclusion}

The UreTron lithotripsy system is a single-handle, singleprobe apparatus that is simple to connect and operate and can generate ultrasound, ballistic lithotripsy, negative pressure suction, and other effects simultaneously, providing safe, effective, and satisfactory lithotripsy results. It has advantages in preventing intraoperative stone displacement, reducing soft ureteroscopy-assisted lithotripsy, improving the stone clearance rate, and reducing postoperative complications, making it worth promoting in ureterolithotripsy.

\section{Ethics and Consent Statement}

This study was conducted with approval from the Ethics Committee of Beijing Chao-Yang Hospital of Capital Medical University. This study was conducted in accordance with the declaration of Helsinki. Written informed consent was obtained from all participants.

\section{Disclosure}

The authors report no conflicts of interest for this work.

\section{References}

1. Scales CD Jr, Tasian GE, Schwaderer AL, Goldfarb DS, Star RA, Kirkali Z. Urinary stone disease: advancing knowledge, patient care, and population health. Clin J Am Soc Nephrol. 2016;11 (7):1305-1312. doi:10.2215/CJN.13251215

2. Raja A, Wood F, Joshi HB. The impact of urinary stone disease and their treatment on patients' quality of life: a qualitative study. Urolithiasis. 2020;48(3):227-234. doi:10.1007/s00240-019-01142-0

3. Raheem OA, Khandwala YS, Sur RL, Ghani KR, Denstedt JD. Burden of urolithiasis: trends in prevalence, treatments, and costs. Eur Urol Focus. 2017;3(1):18-26. doi:10.1016/j.euf.2017.04.001

4. Khoder WY, Bader M, Sroka R, et al. Efficacy and safety of Ho: yAGLaser lithotripsy for ureteroscopic removal of proximal and distal ureteral calculi. BMC Urol. 2014;14(1). doi:10.1186/14712490-14-62

5. Chen S, Zhou L, Wei T, et al. Comparison of holmium: YAG laser and pneumatic lithotripsy in the treatment of ureteral stones: an update meta-analysis. Urol Int. 2017;98(2):125-133. doi:10.1159/ 000448692

6. Purpurowicz Z, Sosnowski M. Endoscopic holmium laser treatment for ureterolithiasis. Cent Eur J Urol. 2012;65(1):24-27. doi:10.5173/ ceju.2012.01.art7

7. Zheng J, Wang Y, Chen B, et al. Risk factors for ureteroscopic lithotripsy: a case-control study and analysis of 385 cases of holmium laser ureterolithotripsy. Wideochir Inne Tech Maloinwazyjne. 2020;15 (1):185-191.

8. Borofsky MS, El Tayeb MM, Paonessa JE, Lingeman JE. Initial experience and comparative efficacy of the UreTron: a new intracorporeal ultrasonic lithotriptor. Urology. 2015;85(6):1279-1283. doi:10.1016/j.urology.2015.03.016

9. Allameh F, Razzaghi M, Fallah-Karkan M, et al. Comparison of stone retrieval basket, stone cone and holmium laser: which one is better in retropulsion and stone-free status for patients with upper ureteral calculi? J Lasers Med Sci. 2019;10(3):179-184. doi:10.15171/ jlms.2019.28

10. Fathelbab TK, Abdelhamid AM, Anwar AZM, et al. Prevention of stone retropulsion during ureteroscopy: limitations in resources invites revival of old techniques. Arab J Urol. 2020;18(4):252-256. doi:10.1080/2090598X.2020.1805966

11. Yan Z, Xie G, Yuan H, Cheng Y. Modular flexible ureteroscopy and holmium laser lithotripsy for the treatment of renal and proximal ureteral calculi: a single-surgeon experience of 382 cases. Exp Ther Med. 2015;10(4):1467-1471. doi:10.3892/etm.2015.2703 
12. Hyams ES, Munver R, Bird VG, Uberoi J, Shah O. Flexible ureterorenoscopy and holmium laser lithotripsy for the management of renal stone burdens that measure 2 to $3 \mathrm{~cm}$ : a multi-institutional experience. $J$ Endourol. 2010;24(10):1583-1588. doi:10.1089/ end.2009.0629

13. Xu CG, Guo YL. Diagnostic and prognostic values of BMPER in patients with urosepsis following ureteroscopic lithotripsy. Biomed Res Int. 2019;2019:8078139.

14. Chugh S, Pietropaolo A, Montanari E, Sarica K, Somani BK. Predictors of urinary infections and urosepsis after ureteroscopy for stone disease: a systematic review from EAU section of urolithiasis (EULIS). Curr Urol Rep. 2020;21(4):16. doi:10.1007/s11934-0200969-2

15. Scotland KB, Lange D. Prevention and management of urosepsis triggered by ureteroscopy. Res Rep Urol. 2018;10:43-49.
16. Patel AP, Knudsen BE. Optimizing use of the holmium: YAG laser for surgical management of urinary lithiasis. Curr Urol Rep. 2014;15 (4):397. doi:10.1007/s11934-014-0397-2

17. Liang H, Liang L, Yu Y, et al. Thermal effect of holmium laser during ureteroscopic lithotripsy. BMC Urol. 2020;20(1):69. doi:10.1186/ s12894-020-00639-w

18. Birowo P, Rasyid N, Atmoko W, Sutojo B. Case report: an occurrence of steinstrasse in retrograde intra renal surgery (RIRS) for large staghorn kidney stone: a difficult experience in managing surgical outcomes. F1000Res. 2020;9:184.

19. Jiang K, Zhang P, Xu B, et al. Percutaneous nephrolithotomy vs retrograde intrarenal surgery for renal stones larger than $2 \mathrm{~cm}$ in patients with a solitary kidney: a Systematic Review and a Meta-Analysis. Urol J. 2020;17(5):442-448.
International Journal of General Medicine

\section{Publish your work in this journal}

The International Journal of General Medicine is an international, peer-reviewed open-access journal that focuses on general and internal medicine, pathogenesis, epidemiology, diagnosis, monitoring and treatment protocols. The journal is characterized by the rapid reporting of reviews, original research and clinical studies
Dovepress

across all disease areas. The manuscript management system is completely online and includes a very quick and fair peer-review system, which is all easy to use. Visit http://www.dovepress.com/ testimonials.php to read real quotes from published authors. 